\title{
GESTIÓN DE SISTEMAS DE INVESTIGACIÓN UNIVERSITARIA EN AMERICA LATINA
}

\author{
Jaim Royero \\ Docente investigador, Instituto Universitario de Tecnología José Antonio Anzoátegui, Venezuela
}

\section{CIENCIA, SOCIEDAD Y UNIVERSIDAD}

Es innegable la relación histórica entre el sistema científico-tecnológico y la sociedad en su conjunto, dado que la actividad científica y tecnológica es concebida como estrategia social para la superación de la pobreza y del atraso social, para Tûnnerman(2002) "el subdesarrollo científicotecnológico es, a la vez causa y consecuencia del subdesarrollo económico-social” (pág.172).

Esta realidad enmarcada en el orden internacional del capitalismo, orienta la modernización y la producción tecnológica de intercambio en las sociedades dominantes, donde la producción, comercialización y transferencia tecnológica, se orienta hacia sociedades poco desarrolladas y dependiente de dichos centros productores. Tal disparidad, hace necesario orientar el desarrollo científico-técnico de estos países hacia una política de fortalecimiento interno capaz de asegurar un lugar en los canales de intercambio tecnológico global.

Bajo esta noción, el carácter sustantivo del desarrollo científico-tecnológico para el avance social, debe orientar toda intención para la construcción de políticas científicas-tecnológicas fomentadoras de la ciencia, de la investigación, de la innovación y la transferencia tecnológica.

En nuestros países, la universidad constituye el principal camino para el fortalecimiento de las estructuras nacionales de producción científica, ya que en ellas se concentran en gran medida los resultados alcanzados sobre innovación y desarrollo de conocimientos científicos, así como la infraestructura necesaria y el personal calificado para el diseño y ejecución de lineamientos estratégicos de carácter nacional.

\section{SOCIEDAD Y EDUCACIÓN SUPERIOR}

El papel de las universidades y muy específicamente el sistema de educación superior, tiene sin duda una responsabilidad ante la sociedad en su conjunto, ya que esta última exige a la universidad producir, entre otras cosas, conocimiento científico socialmente válido capaz de generar soluciones creativas en las múltiples áreas del quehacer social. Estas exigencias según Royero(2002), se perfilan por las siguientes razones socio políticas: 
?? La pérdida de calidad y efectividad social de estas instituciones que ponen en alerta al ente financista de las misma, el Estado, que a su vez es responsable de dicha crisis.

?? La acentuada crisis de los sistemas de planificación educativa como acción correctiva de los sistemas institucionales.

?? La dualidad y contradicción de la organización del sistema de educación universitario.

?? La herencia política indiferente y cómplice a la crisis del sector.

?? La asignación presupuestaria irrisoria.

?? La intención de control total por parte del Estado obstaculizadora de la necesaria autonomía.

?? La creciente demanda de los demás sistemas sociales a la educación como símbolo de desarrollo.

?? La ausencia de una cultura de la planificación y dirección sistemática de estas organizaciones.

?? La utopía de la masificación versus la calidad

?? La necesidad de mejorar los sistemas institucionales.

Sobre la base de estas exigencias, "las funciones de la universidad adquieren también una nueva dimensión frente a la revolución socio-científica, provocada por el papel central de la ciencia en la sociedad post-industrial” (Tûnnermann, 2002, pág. 96).

Dicha dimensión proviene de la necesidad de llevar el conocimiento a todas las esferas de la sociedad, de actualizar el conocimiento debido al vertiginoso avance del desarrollo social y a la aplicación de dicho conocimiento como parte de una estrategia de desarrollo nacional en los países latinoamericanos. Esta realidad, hace que las instituciones de educación superior requieran cambios sustanciales en sus sistemas de investigación y gestión tecnológica.

\section{EL SISTEMA DE INVESTIGACIÓN UNIVERSITARIA EN AMÉRICA LATINA}

La realidad latinoamericana sobre los resultados obtenidos en los sistemas de investigación universitarios, tiene sin duda una noción muy particular en lo referido a la producción, apropiación y aplicación de conocimientos y tecnologías. Cada país del continente tiene su propia visión sobre la gestión de la investigación y los resultados alcanzados por los esfuerzos desarrollados por estos países se desconocen a grandes rasgos. Hasta el presente, no se dispone de los datos necesarios para evaluar la situación actual y las perspectivas de los sistemas encargados de formar recursos humanos y de impulsar la investigación científica en la 
región(UNESCO,1999), a pesar de esto, los trabajos de Lemasson y Chippe ${ }^{1}$ (1999) dan una aproximación valiosa a la realidad sobre la investigación universitaria en América Latina.

Estos autores realizaron un estudio de casos tomando en consideración a países como Argentina, Brasil, Chile, Colombia, México, Uruguay y Venezuela, sobre elementos de política de ciencia y tecnología, mecanismos institucionales de apoyo a la investigación, características de los sistemas e instituciones de educación superior, financiamiento del sector universitario e importancia relativa de las universidades en la investigación.

En el caso Argentino, los organismos encargados de la gestión de la investigación recaen en la Secretaria de Estado de Ciencia y Tecnología, el Consejo Nacional de Investigación en Ciencia y Técnica(CONICET) y la Secretaria de Política Universitaria(SPU). Las instituciones de educación superior nacionales tienen gran relevancia en los resultados de investigación en dicho país, la organización de estas se concentran básicamente en unidades administrativas o secretarias orientadas a la producción científica. En la Universidad Nacional de Córdoba, la Secretaria de Ciencia y Tecnología, en la Universidad Nacional del Rosario opera la Secretaría de Ciencia y Técnica, y en la Universidad Nacional de Mar de Plata, la secretaría de Ciencia y Técnica, entre otras.

Con respecto a Brasil, la dirección de la investigación científica recae en el Consejo Nacional de ciencia y Tecnología y el Ministerio de Ciencia y Tecnología. En relación a Chile el CONICYT representa la unidad asesora y de financiamiento de la investigación nacional cuyo apoya es representado por el FONDECYT y el FONDEF. En Colombia El Consejo Nacional de Política Económica y Social(COMPES) y el COLCIENCIAS adscrito al ministerio de planeación, constituyen los organismos principales de gestión y financiamiento de la investigación.

En el caso mexicano, la Secretaría de Educación Pública (SEP) y el Consejo Nacional de Ciencia y Tecnología(CONACYT) son los órganos principales de diseño y ejecución de políticas de investigación. Las universidades nacionales comparten la producción científica con unidades administrativas como la Coordinación de la Investigación Científica $(\mathrm{CIC})$ dependiente del Consejo Técnico de la Investigación Científica(CTIC) de la Universidad Autónoma de México. En la Universidad autónoma de Chapingo dirige el sistema de investigación la Dirección General de Investigación y Postgrado a través de la subdirección de Investigación, entre otras.

En el caso uruguayo, estos autores destacan la particularidad de este país al no poseer organismos encargados de gestionar y dirigir una política científica estatal, los resultados de investigación se caracteriza por la dispersión de los mismos, tal es el caso de la Universidad de la

\footnotetext{
${ }^{1}$ Para una revisión exhaustiva véase Jean Pierre Lemasson y Marta Chiappe. La investigación universitaria en América Latina. Ediciones IESALC/UNESCO, Caracas, 1999.
} 
República, el Instituto Nacional de Investigaciones Agrícolas, El Instituto de Investigaciones Biológicas Clemente Estable y el esfuerzo privado representado por los Centros de Investigación en Ciencias Sociales(CCSS). También destaca El Consejo Nacional de Investigaciones Científicas y Técnicas(CONICYT) encargado sólo de promover y estimular el desarrollo de investigaciones a escala nacional.

En el caso venezolano, el desarrollo científico técnico experimentó a partir de los años 60 y 70 una concepción denominada modelo" populistaestatista-rentista de la investigación. Populista por que se proponía una expansión y ampliación del acceso a la investigación equivalente a la que se daba en la educación; estatista porque su financiamiento provenía en la casi totalidad del Estado; y rentista porque se recibía sin responsabilidad clara de contraparte por parte de los grupos y centros de investigación científica" (Yero,1993)

A partir de la década de los 80 , en el país se inicia un deterioro económico-social en torno a la capacidad productiva y el financiamiento de la investigación, se inicia una nueva etapa llamada utilitaria-selectiva-competitita rentable-privatizada"(Idem). El esfuerzo del Estado se concentró en desarrollar una sociedad innovadora y productora de nuevas tecnologías para el desarrollo industrial de la nación. Para Díaz y Vessuri(1982) este proceso se caracteriza por ser de carácter utilitarista, es decir, las ciencias como sinónimo de tecnología bajo la exigencia social de resolver problemas puntuales.

La crisis económica que caracterizó este periodo determinó cambios sustanciales del sistema de educación superior. La reducción sustancial del gasto público como estrategia para el pago de la deuda nacional, obligó a una reducción drástica en la inversión de la educación y al sector de ciencia y tecnología(Lemasson y Chiappe,1999).

En el año 1994,Venezuela destinaba un $0,35 \%$ de su PBI para investigación y desarrollo, el porcentaje más bajo desde 1990, la educación superior para 1995 contribuye con un $22 \%$ del gasto en investigación.(Idem)

En Venezuela los organismos públicos encargados de la gestión tecnológica se concentran en el Ministerio de Ciencia y Tecnología y el Consejo Nacional de Investigaciones Científicas y Tecnológicas(CONICIT) creado en 1967 y adscrito a dicho ministerio .Dentro de sus programas más importantes, el CONICIT conjuntamente con el BID iniciaron hasta 1997 un convenio para el financiamiento de proyectos de investigación, infraestructura tecnológica, capacitación del recurso humano y transferencia tecnológica. 
De igual modo el CONICIT crea el programa Sistema de Promoción del Investigador( PPI) como estrategia para el incentivo de los investigadores del país en los sectores universitarios ,públicos y privados. Igualmente desarrolla el programa del investigador novel como mecanismo de desarrollo de una generación de relevo capaz de producir conocimientos en las distintas áreas de conocimiento.

Para Vessuri(1996) el PPI responde a una exigencia de los sectores académicos nacionales por el reconocimiento social de la actividad investigativa y por ende del estímulo necesario ofrecido a los investigadores del país nacional.

En lo referente a la educación superior, el sistema está conformado por las Universidades nacionales autónomas, experimentales y privadas, y por instituciones no universitarias concentradas en los institutos de tecnología y colegios universitarios. El sector universitario nacional es coordinado por el Consejo Nacional de Universidades (CNU) y apoyado por la Oficina de Planificación del Sector Universitario (OPSU) igualmente, el Ministerio de Educación Superior de nueva data, conforman las estructuras básicas de gestión de la educación superior en el país.

Para 1995 el 73,5\% de los integrantes del PPI eran docentes ubicados en las universidades nacionales, dentro de las mismas, las áreas dominantes se concentran en Ciencias Médicas, Biológicas y del Agro con un 37,4\%, le siguen las Ciencias Físicas, Químicas y Matemáticas con un $24 \%$, con un $20 \%$ las áreas de Ciencias Sociales y un $18,6 \%$ en las áreas de Ingeniería, Tecnología y Ciencias de la Tierra. En el sector público nacional, el 56,8 \% de los investigadores se encuentran en las áreas de Ciencias Médicas Biológicas y del Agro, mientras que el 43,2\% restante se ubican en las otra áreas mencionadas(Vessuri,1996).

Con relación a las publicaciones, entre el periodo que va desde 1986 hasta 1991, se publicaron 2000 artículos científicos en revistas arbitradas, lo cual colocó al país en el quinto lugar como productor de conocimiento científico y tecnológico de América Latina. La mayoría de dichos artículos provienen del Instituto Venezolano de Investigación Científica seguidos de tres universidades nacionales como lo son la Universidad Central de Venezuela, la Universidad Simón Bolívar y la Universidad de los Andes(Lemasson y Chiappe,1999).

En conclusión, la realidad latinoamericana en torno a la gestión y producción de sus sistemas nacionales de investigación universitarias, arroja resultados pocos alentadores en torno a la inversión en investigación y desarrollo, dicho estudio muestra que "ningún país sobrepasó el $1 \%$ del PBI... el porcentaje promedio de América Latina para el año de 1992 estuvo en el orden del 0,4\%, en comparación con el 1,4\% en Italia o Canadá en 1991, y en 1994 sobrepasó el 2,0\% en países como Francia(2,3\%), Estados Unidos (2,54\%) o Japón con un 2,73 \%. Brasil y México 
representan las tres cuartas partes del total de los fondos invertidos y dominan ampliamente en el continente, pero en comparación con el mundo industrializado ambos se encuentran por debajo".(Idem)

Por otra parte, "es el hecho de que, excepto Brasil, los sistemas de educación superior han evolucionado independientemente de los sistemas nacionales de ciencia y tecnología. Los organismos nacionales de ciencia y tecnología mantuvieron las tradicionales políticas gubernamentales de corto plazo, sin lograr introducir en las grandes políticas la coordinación de la formación de recurso humanos de alto nivel, la actualización permanente de la infraestructura para investigación científica, el estímulo a la creación de grupos de trabajo y la generación de "masas críticas" de investigadores en determinados temas prioritarios".(Idem)

Como se nota a grandes rasgos, la investigación científica en América Latina requiere un replanteamiento en torno a la articulación armónica entre universidad, Estado y sistemas de ciencia y tecnología. Esto con el fin de repensar el papel de la ciencia en un mundo desproporcionado desde el punto de vista económico-social.

La investigación científica en las instituciones de educación superior se enfrenta a grandes retos como lo es la insuficiencia de recursos para el sector, el empobrecimiento de la actividad científica la cual orienta a la fuga de cerebros hacia el centro de producción desarrollados y privados, y la marcada desorganización institucional para la construcción de una política integral de desarrollo tecnológico latinoamericano autónomo donde se incluyan sistemas de gestión, control y evaluación.

\section{LA EVALUACIÓN DE CENTROS DE INVESTIGACIÓN Y DESARROLLO}

En la mayoría de las instituciones de educación superior de América Latina no se ha desarrollado una cultura de la evaluación. Prevalece una racionalidad interna de auto reproducción basadas en decisiones burocráticas y corporativas, sin una función de evaluación, y sin un juicio externo respecto a los fines, eficacia, eficiencia, capacidad, pertinencia y calidad de los servicios básicos que ofrece sus actividades académicas (UNESCO,1997)

En referencia a la necesidad de contar con modelo de evaluación institucional para los sistemas universitarios, González y Ayarza (1997) realizan una taxonomía sobre los modelos de evaluación de la calidad en educación superior, describen el modelo sistémico desarrollado por autores especializados como Stufflebeam (1974), Astin (1974) y Kuh (1981) sustentado en el principio del enfoque de sistemas. 
Dicho modelo reconoce que las instituciones universitarias son un sistema dentro de un sistema más complejo y dinámico, llámese sistema educativo y sistema social, de igual modo reconocen la visión abierta de estas instituciones al caracterizar las mismas en un proceso de intercambio permanente y constante con el ambiente externo.

Estos autores presentan lo dinámico de las instituciones universitarias en torno a la relación que tiene el ambiente con las entradas (información, energía, recursos, materiales) del sistema y su transformación a través de procesos institucionales regulatorios, operativos o de apoyo, con el fin de obtener salidas o productos que interactúan con su entorno. Dichos elementos requieren procesos de retroalimentación y control constantes para mantener y equilibrar el sistema institucional.

Por otra parte, describen el modelo globalizado de Robert Stake que comprende la descripción de los componentes de la evaluación en: la evaluación del esfuerzo de la energía puesta y los resultados obtenidos, la evaluación de la efectividad vista como la relación entre los objetivos logrados y los resultados obtenidos, la evaluación de la eficiencia relacionada con el uso racional de los recursos; la evaluación de los procesos y la evaluación de la relevancia.

Un tercer tipo de modelo decrito por estos autores, es el llamado modelo etnográfico definido como "el proceso de proveer una descripción científica de sistemas educacionales, procesos y fenómenos dentro de su contexto específico".

Un cuarto modelo más elaborado y aplicado en América Latina es el realizado por el Centro Interuniversitario de Desarrollo (CINDA) que ejecuta el proyecto de gestión y evaluación universitaria, integrado al programa multinacional de educación media y superior de la OEA.

Dicho modelo se sustenta en los aportes teóricos realizados pos Stake, las observaciones etnográficas de Spradley y la Teoría de sistemas. El modelo identifica seis dimensiones básicas para medir la calidad como lo son: la relevancia, efectividad, disponibilidad de recursos, eficiencia, eficacia y procesos, así como los indicadores, la función universitaria que involucra, el nivel en que se aplica y la fuente de donde se recogen los datos provenientes de los actores participantes en el proceso.

En una visión más específica relacionada con los sistemas de investigación, Licha(1993) describe a grandes rasgos el modelo de Stepan para el desarrollo de instituciones de investigación. Dicho modelo se concentra en el estudio de la organización, sus objetivos, funciones y recursos, el papel de sus líderes, el equipo de formación, los programas de investigación pertinentes y la actitud docente y de extensión de los grupos de investigación. 
Por otro lado, refiere el modelo de Stolte-Heiskanen el cual plantea la evaluación de tres procesos básicos: la œntribución de los centros de investigación al avance del conocimiento, el prestigio del grupo que lo produce y la efectividad del esfuerzo en cuanto a: El impacto en la generación del nuevo conocimiento, el impacto en la formación científica técnica del entrenamiento de los investigadores, el valor social de conocimiento producido y aplicado, el desarrollo experimental y el éxito del cumplimiento del programa de investigación.

En un enfoque más administrativo, Peña (1998) describe los modelos de evaluación desde el punto de vista de la definición propia de la organización aplicable a centros de investigación, estos son: La planificación estratégica, la evaluación del desempeño organizacional, la evaluación de los sistemas de movilización de recursos, la evaluación de los procesos gerenciales, el desarrollo organizacional., la evaluación de la cultura organizacional y el modelo estructurafuncional.

\section{LA PLANIFICACIÓN ESTRATÉGICA COMO MODELO DE EVALUACIÓN DE LA GESTIÓN DE LA INVESTIGACIÓN UNIVERSITARIA}

Dentro de la universidad latinoamericana se ha adoptado una consideración con relación a la aplicabilidad de modelos de evaluación más eficientes y racionales, para Tûnnermann(2000), la educación superior ha adoptado una visión más prospectiva en la evaluación de sus escenarios a largo plazo donde el planeamiento estratégico se ha incorporado a la gestión y dirección de la universidad latinoamericana.

La planificación estratégica en instituciones de educación superior, provino del campo de la teoría administrativa de la gerencia privada, ella se destacó gracias a los trabajos de Ackoff, Porter y Steiner, los cuales destacaron la interpretación y desarrollo del llamado modelo estratégico corporativo, el cual plantea que el ejercicio planificativo se centra en los supuestos que la organización constituye una corporación en intenso intercambio con su ambiente o entorno, donde participan actores o competidores con capacidad de desequilibrar o potenciar las estrategias basadas en las fortalezas y debilidades que estas puedan tener en un medio complejo y dinámico.

En este proceso la acción planificadora se centra en un primer nivel llamado corporativo, en donde a partir del análisis de entorno y el análisis interno, se desarrolla la filosofía institucional. En un segundo nivel llamado funcional, se desarrollan los planes funcionales caracterizados por su detalle en la configuración de los objetivos y metas tácticas a ejecutar por la organización a través de los programas y proyectos, y por último, un nivel denominado operacional basado en la 
ejecución de los programas por medio de los planes operativos con su vinculación respectiva al presupuesto.

En síntesis, la planificación estratégica constituye, en palabras de Serna (2000) "el proceso mediante el cual quienes toman decisiones en una organización obtienen, procesan y analizan información pertinente, interna y externa, con el fin de evaluar la situación presente de la empresa, así como su nivel de competitividad con el propósito de anticipar y decidir sobre el direccionamiento de la institución hacia el futuro "( pág. 17).

La anterior definición precisa tres premisas esenciales en la comprensión del concepto: una primera donde ubica al proceso de planificación estratégica como acción de agentes decisorios mayormente ubicados en el nivel estratégico e interrelacionados con los niveles coordinativos y operativos. Una segunda premisa la cual ubica a la planificación estratégica como una actividad donde la abstracción de la realidad interna y externa de la organización, impulsa la elaboración de un tercer elemento basado en el desarrollo de futuros deseables y posibles, a fin de avizorar en perspectiva, las oportunidades de un entorno lleno de incertidumbres.

Estas apreciaciones hacen de la planificación estratégica un sistema metodológico estructurado por procesos técnicos e instrumentales explicados sucintamente en los párrafos subsiguientes.

\section{EL PROCESO DE PLANIFICACIÓN ESTRATÉGICA}

La planificación estratégica como sistema contiene un determinado número de procesos que hacen de ella una actividad dinámica, flexible y continua. Esta complejidad no viene dada por las dificultades propias del proceso en torno a la aplicación de sus métodos y técnicas, sino más bien corresponde a la dinámica de la realidad que desborda los más elaborados análisis del entorno y hacen de esta, un instrumento racionalizador de las aspiraciones organizacionales en un mundo de incertidumbres constantes.

La planificación estratégica asume un rol importante en la construcción de escenarios factibles dentro de cierta certidumbre esperada, para ello, establece un conjunto de procesos integrados capaces de sistematizar acciones que permitan lograr dentro de un contexto específico, objetivos y metas productivas.

Para poder comprender los pasos básicos de la planeación estratégica Koonts y Weihrich (1996) proponen un esquema concreto del proceso: 
?? Identificación de oportunidades y amenazas: Consiste en el estudio del entorno bajo premisas de actuación de la organización en un mundo de oportunidades y amenazas en concordancia con el estudio interno de sus debilidades y fortalezas para hacer frente a dicho entorno.

?? Especificación de objetivos: Esta fase tiene como propósito diseñar los objetivos de forma jerárquica en las distintas unidades que conforman la organización.

?? Determinación de las premisas: Las premisas son las suposiciones sobre el ambiente en el que el plan debe realizarse. Define el análisis de las contingencias del medio interno y externo a la organización para cumplir sus objetivos.

?? Detección de los cursos de acción alternativos: Refiere la búsqueda de alternativas o estrategias más pertinentes para el logro de los objetivos a lograr.

?? Evaluación de alternativas: Luego de escoger las alternativas más viables, éstas se valoran en torno a su claridad en los costos y resultados que puedan esperarse de su aplicación y se jerarquizan a fin de tomar las más efectivas para el éxito organizacional.

?? Implantación: Supone la utilización de los recursos materiales, humanos y financieros para aplicar la alternativa elegida.

?? Control: A través de indicadores relevantes y prediseñados, se evalúan los resultados parciales que se vayan logrando y se procede a la revisión del proceso a fin de detectar los posibles errores que impidan el logro de los objetivos establecidos.

Dentro de su configuración final, la planificación estratégica realiza los mismos pasos anteriormente descritos en los niveles coordinativos y operativos, es decir, cada unidad coordinadora y operativa deberá diagnosticar, definir estrategias, proyectos y planes concretos que provengan de la filosofía organizacional a fin de llevar a cabo acciones pertinentes para el logro de los objetivos propuestos a partir de sistemas de control de gestión capaces de garantizar la eficiencia de los recursos invertidos y el alcance de las metas.

Bajo esta perspectiva, el control de gestión constituye un proceso esencial para la realización de actividades de planeación estratégica ya que constituye el camino para la evaluación de las ideas y acciones definidas en el proceso anterior, por lo que su definición, características, modelos, categorías y conceptos, se desarrollarán ampliamente en la siguiente parte del trabajo. 


\section{EL CONTROL DE GESTIÓN COMO HERRAMIENTA ADMINISTRATIVA PARA LA DIRECCIÓN DE SISTEMAS DE INVESTIGACIÓN UNIVERSITARIOS}

\section{El control como proceso}

La concepción de la organización universitaria como sistema social abierto, involucra la configuración de subsistemas capaces de dirigir las instancias mediante las cuales las organizaciones se crean. Los sistemas de gestión constituyen un elemento fundamental para la orientación de los esfuerzos y recursos que se le inyectan a cualquier sistema social, incluidas las instituciones de educación superior. Estos esfuerzos se validan por medio de las funciones o procesos administrativos como lo son la planeación, organización, mando, ejecución, control y evaluación.

La planeación vista en la parte anterior, refiere la dirección del sistema a través del estudio de su entorno y la especificación de políticas, estrategias y proyectos para la consecución de sus fines.

La organización hace referencia al diseño y agrupación de tareas, la asignación de responsabilidades y el establecimiento de mecanismos de comunicación entre las distintas unidades administrativas.

El mando tiene que ver con la manera en que se toman las decisiones y propiciar la participación a través de procesos de liderazgo, trabajo en equipo, motivación, etc. , a partir de su ejecución en el contexto organizacional.

En cuanto al control, este regula las desviaciones que pueden presentar los sistemas por medio de procesos que permiten medir y corregir tales desviaciones en un tiempo y espacio determinado.

En torno a la evaluación, esta representa la retroaliment ación del sistema ya que se miden los resultados de la gestión en relación con los objetivos propuestos. Esta relación estrecha entre estos procesos, hace que la gestión universitaria sea una acción sistémica, armónica y compleja, donde la regulación y el seguimiento constituyan procesos claves para la eficacia del sistema.

La importancia del control en estos términos de complejidad, radica en el impacto que produce en los propios objetivos, ya que de su aplicación puede revertirse o adaptarse el sistema 
de objetivos organizacionales, provocando cambios sustanciales en la asignación presupuestaria en el propio sistema social y en la eficiencia del proceso de administración universitaria.

En estos términos, el control ha sido definido bajo dos grandes perspectivas a partir de la teoría administrativa, una perspectiva limitada y una perspectiva amplia. ${ }^{2}$ Desde una perspectiva limitada, el control se concibe como la verificación a posteriori de los resultados conseguidos en el seguimiento de los objetivos planteados y en el control de gastos invertido en el proceso realizado por los niveles directivos donde la estandarización en términos cuantitativos, forman parte central de la acción de control.

Bajo una perspectiva amplia, el control es concebido como una actividad no sólo del nivel directivo, sino de todos los niveles y miembros de la entidad, orientando a la organización hacia el cumplimiento de los objetivos propuestos bajo mecanismos de medición cualitativo y cuantitativo. Este enfoque pone énfasis en los factores sociales y culturales presentes en el contexto institucional ya que parte del principio que es el propio comportamiento individual quien define en última instancia, la eficacia de los métodos de control elegidos en la dinámica de gestión.

Para efectos de este análisis, se concibe al control como el mecanismo que permite corregir desviaciones a través de indicadores cuantitativos y cualitativos dentro de un contexto social amplio, a fin de lograr el cumplimiento de objetivos claves para el éxito organizacional, es decir, el control se entiende no como un proceso meramente técnico de seguimiento, sino también como un proceso informal donde se involucran factores culturales, organizativos, humanos y grupales.

\section{EL PROCESO FORMAL DE CONTROL}

La estructuración del proceso de control, según Pérez (2000), ya sea técnico e informal, requiere la consideración de los siguientes elementos:

a. Centros de responsabilidad: Estos constituyen las unidades o sujetos pertenecientes a la estructura organizacional donde la definición de funciones, responsabilidades y tareas, son la base para definir el grado de responsabilidad en las decisiones y seguimiento de las tareas para el control, en concordancia con las estrategias y objetivos establecidos por la organización.

\footnotetext{
${ }^{2}$ Para una explicación más rigurosa Véase Amat Joan: El control de gestión(Una perspectiva de dirección Ediciones Gestión 2000, Barcelona,2000, pág.27
} 
b. Las actividades del control: Constituyen las distintas fases mediante el cual se aplica el proceso en la actividad de dirección, es decir, la fijación de objetivos, el establecimiento de estándares e indicadores, la comparación de resultados, las correcciones estimadas y la evaluación en su práctica.

c. Un sistema de información: Constituye la plataforma de datos, análisis de datos y salidas de información necesaria para la administración del sistema de control.

En el ámbito organizacional, el control como proceso del sistema de gestión es un instrumento necesario para la eficacia organizacional, debido a la constante incertidumbre y a la propia escasez de los recursos. Desde su ámbito primario, el control pasó a constituirse en un sistema capaz de asegurar una efectiva gestión de la totalidad del sistema institucional, acuñado en la administración moderna e insertado en las actividades de los directivos más exitosos de hoy.

\section{EL CONTROL DE GESTIÓN}

Definir el concepto de control de gestión implica considerar el desarrollo del mismo en el ámbito administrativo, distintos autores han definido el concepto de acuerdo a sus propias posiciones e interpretaciones, sin embargo, es preciso describir cuál ha sido su evolución teórica en el transcurso de las últimas décadas a fin de destacar su impacto en el desarrollo organizacional.

Dentro de la descripción y valoración del control de gestión se especifican dos concepciones comúnmente aceptadas en el ámbito administrativo, por un lado se tiene al control como necesidad inherente al proceso de dirección(enfoque racional) y por el otro, en un paradigma más integral vinculado no sólo a la dirección formal, sino a factores claves como la cultura, el entorno, la estrategia, lo psicológico, lo social y la calidad, representados por los llamados enfoques psicosocial, cultural, macrosocial y de calidad.

El enfoque racional se centra en la concepción del control como mecanismo determinante y sistemático, cuya implantación permite lograr por sí solo la eficiencia y eficacia de la organización. Este enfoque defendido por la escuela clásica, se evidencia por una particular definición dada por Fayol en su conocida obra llamada Administración Industrial y General, señalando que el control "consiste en verificar si todo se realiza conforme al programa adoptado, a las órdenes impartidas y a los principios admitidos" con el fin de detectar posibles desviaciones evitando su repetición. Esta concepción simple orientada a la excesiva formalización de los mecanismos de control se fortalece con los trabajos de Anthony(1965) en torno a entender el proceso de control por centros de responsabilidad y la medición de variables por medio de indicadores ligados a los aspectos claves 
de la organización, este enfoque, en palabras de Amat(2000) constituye una visión" excesivamente técnico y racional del proceso de control al limitarlo al diseño de aspectos formales reduciendo la complejidad del contexto organizativo en el que aquel opera”( pág.50.)

Siguiendo al análisis realizado por este autor dentro del enfoque racional, el concepto de control se desarrolla al introducir cálculos matemáticos (Abdel-Khalik y Lust,1974; y Kaplan,1982) en el tratamiento de los precios de transferencia en lo que se conoce como la teoría de la agencia (Demski.1983; Baiman y Demski,1980). La teoría de la agencia entiende al control como el establecimiento de un contrato óptimo entre los objetivos individuales(responsables de la unidad y de la organización) por medio de la aplicación del análisis microeconómico del control.

Dentro de esta corriente, Amat ubica la teoría de la contingencia(Thompson,1967 y Woodward,1965) como representación del enfoque racional. La teoría de la contingencia entiende al control como el ajuste de la tecnología, la dimensión y e entorno a la eficacia de cómo se enfrenten las contingencias vividas por la organización. Del mismo modo critica tal postura al afirmar que en ella" las relaciones de las variables no están suficientemente claras; no se ha estudiado la relación control-eficacia y no se ha tenido suficiente contrastación empírica”3

Superando lo aportes del paradigma racional, a partir de los años 50 y 60 el paradigma psicosocial entendió al control como un proceso fundamentado en la consideración de aspectos motivacionales y conductuales al momento de implantar modelos de control organizacional, estos principios fueron desarrollados por la Escuela de las Relaciones Humanas, El Procesamiento Humano de la Información y la Teoría de Sistemas.

La Escuela de las Relaciones Humanas planteó la influencia que tenía la aplicación de sistemas de control en los individuos, por lo que la participación de éstos en el proceso de decisión y control, contribuirían al aumento de la motivación al relacionar los objetivos individuales con las metas organizacionales, al respecto son fundamentales los trabajos de Argyris,(1952), Searfoss(1976), Lewin(1970), Merchant(1986), Hopwood(1972), De Coster y Fertakis(1968),entre otros.

La Escuela del Procesamiento Humano de la Información se centra en entender al control como la aplicación de los avances de la psicología cognitiva para el estudio de los elementos que inciden en el comportamiento y conducta en torno a las decisiones de los empleados a la concepción del control, estos elementos se basan en las características de la información, los procesos cognitivos y las decisiones personales, al respecto se tienen los trabajos de Libby y Lewis(1982), Prakash y Rappaport(1977) y Driver y Mock,1975).

\footnotetext{
${ }^{3}$ Véase las críticas al paradigma racional en Amat, Joan. ob.cit. Pág.51.
} 
Estos aportes, según Amat al concepto de control, se orientan a la desconsideración de aspectos sociales y organizacionales, centrándose solamente en elementos de carácter conductual y comunicacional, del mismo modo no sustenta el papel de la distribución del poder y la influencia de enfrentar las incertidumbres organizacionales(véase los trabajos de Hopwood,1978) y evita el no consentimiento de otros aspectos que no sean los cognitivos(Critica de Sweringa y Weick, 1982).

En torno al enfoque de sistemas, éste albergó una nueva idea acerca del papel del control dentro de la organización. Dicha idea se centraba en la definición de dos principios básicos como lo son la regulación y el control insertados por la cibernética. Para Bertalanffy, la cibernética es la teoría de los mecanismos de control en la tecnología y en la naturaleza, fundada en los principios de la información y retroalimentación. En esta corriente, los sistemas intercambian información y energía con su entorno, la evalúan y reorganizan sus componentes sobre la base del control que actúa como ente regulador de los fines del mismo. Estas ideas fueron introducidas en la organización por medio de los trabajos de Katz y Kahn(1978) y profundizados por el modelo sociotécnico de Tavistok.

Sin embargo, este enfoque ha sido criticado con suficientes razones sobre la base de las propias limitaciones del proceso de control, al respecto, Amat señala que los conceptos provenientes de la biología dificultan la consideración y aplicación a la organización ya que la mayoría de sus conceptos provienen de la cibernética como ciencia no social.

En otro orden de ideas, en la década final de lo 70 y principios de lo 80 , la perspectiva del control se enfocó hacia la consideración de la cultura como variable importante de los sistemas de control, al respecto Young(1979), Collins(1982),Flamholtz(1983) Hosfstede(1987), Dent(1986) y Dermer y Lucas, trabajaron sobre las bases de incorporar el concepto de cultura como mecanismo capaz de orientar procesos de control mas efectivos, dado que el individuo como ser social, comparte valores, ritos y creencias que pueden facilitar la efectividad de los sistemas de control en la organización. En perspectiva, tal enfoque aportó un elemento importante para el estudio del control, pero obvió el rol de los valores sociales desarrollados por el sujeto en su entorno familiar y social, en sus explicaciones teóricas.

A finales de los años 80, la perspectiva macrosocial constituyó un desarrollo novedoso en la interpretación del control, ya que por un lado aceptaba el papel de la cultura en la efectividad de los procesos de control, pero por el otro agregaba la fuerza del entorno sociocultural y político dada la creciente incertidumbre del sistema económico y su impacto en las organizaciones. 
Al respecto dicho modelo enfoca dos corrientes fundamentales, la "cross culturalcrossnational" y el enfoque radical". La primera considera el entorno como una variable externa de la organización y la influencia de la cultura de cada país en los sistemas de control, debido a las características socio culturales de la empresa y sus miembros, al respecto se tienen los trabajo de Beres y Portwood(1981), Hofstede(1983) y Horowist(1979). La corriente radical critica todas las aportaciones anteriores intentando incorporar al control como resultado social y cultural dentro de una perspectiva socio-política de contradicciones sociales, véase trabajos de Tinker,Merino y Neimark(1982-1986).

A pesar de que los enfoques socio culturales han aportado fundamentos interesantes en la concepción del control, se ha podido vislumbrar en la década de los 90, una tendencia hacia la concepción tradicional de medición de indicadores pero apoyado en novedosos sistemas de información e informática, al respecto, el modelo de McNair, Lynch y Cross(1990) se centra en la consideración de tres niveles básicos: las unidades de negocio, el área corporativa y los centros de actividad de los departamentos. Los centros de actividad y departamentos son el corazón de esta pirámide de resultados; de hecho. Se basan en los conceptos de Peingeniería(entrega, tiempo ciclos), calidad y ABC(sobrecoste-pérdidas) en su base.

En torno al nivel operativo, se trata de un nivel relacionador, es decir, los objetivos definidos se basan en criterios como la satisfacción de la clientela, flexibilidad y productividad, vinculados tanto con las metas establecidas en el nivel inferior(departamentos, procesos y actividades) como en el inmediatamente superior(unidades de negocio)configurándose lo que se denomina "income drivers"(inductores de resultados) de los objetivos de ese nivel superior.

Otra propuesta es la de Sveiby(1995) es un modelo que trata de medir los activos intangibles y ofrece un sencillo formato para la visualización de los indicadores más relevantes. La estrategia de la organización resulta relevante para la determinación de dichos indicadores. En este modelo, las partes más importantes a cubrir son crecimiento, cambio, renovación, eficacia y estabilidad, debiéndose configurar un par de indicadores para cada criterio, igualmente, puede observarse el modelo SIGER(Sistema Integrado de Gestión de la Estrategia) desarrollado por .López y Hernandez (1999), basado en la definición de indicadores a partir de las estrategias y objetivos de la organización.

En otro enfoque más moderno, la Fundación Europea para la dirección de la calidad, propone un modelo de gestión integrada sobre la base de la visión globalizada y enfocada en el cliente a partir de los principios fundamentales de la calidad total. El concepto de control bajo la perspectiva de calidad, incluye un conjunto de herramientas y técnicas de control de los procesos 
básicos de la organización; entre ellas se tiene al control estadístico de procesos(SPC), el control de la calidad total(TQC) y la gestión de la calidad total (TQM).

Com o se apreció en los párrafos anteriores, son muy diversas las interpretaciones sobre el proceso de control en la organización empresarial. En el ámbito público, estos modelos han sentado la base para su aplicación en el contexto universitario a nivel mundial, la estrecha relación que guarda hoy la administración como ciencia y la necesidad de la educación superior de contar con sistemas de dirección propios acordes a sus realidades, hace que se desarrollen en el continente conceptos y categorías de dirección estratégica en el ámbito universitario aplicados a las mejoras institucionales de los procesos de docencia, investigación, extensión y servicios.

\section{CONTROL DE GESTIÓN EN INSTITUCIONES DE EDUCACIÓN SUPERIOR}

En torno al control de gestión en instituciones de educación superior, este se ha caracterizado por la aplicación de controles presupuestales a razón de las exigencias gubernamentales de seguimiento de gastos en educación y a las normas de control financiero asumidas por dichas instituciones en el ámbito público.

Estas exigencias comenzaron a prevalecer rotundamente a mediados de los años 80 y principio de los 90 a raíz de la racionalización del gasto y a la crisis económica de la mayoría de los países de la América Latina. El nuevo esquema "racionalizador" del gasto obligó a los gobiernos a fortalecer los controles en la distribución y asignación de recursos al sector.

Dicha situación obligó prácticamente a las universidades a diseñar mecanismos formales e integrales de planeación institucional. El planeamiento universitario es reciente en la mayoría de las universidades nacionales en Latinoamérica, para Tûnnermann (2002) la función del planeamiento ha sido vista como algo importante y necesario para la gestión universitaria, aunque todavía esta situación corresponde a un número muy limitado de universidades.

Del mismo modo, considera que una de las características de la universidad latinoamericana tradicional, es la ausencia de una organización administrativa eficaz y el desconocimiento de la importancia de la administración académica y de la administración de la ciencia. Estas características han impacto igualmente en la gestión de sistemas de investigación en Latinoamérica, al respecto Lemasson y Chiappe(1999) afirman entre otros puntos, que dicha realidad se caracteriza por las siguientes situaciones: 
?? Insuficiencia en las actividades de investigación y la ausencia de mecanismos para el desarrollo de la investigación en la mayoría de las universidades latinoamericanas.

?? La mala articulación entre los entes de ciencia y tecnología de carácter estatales y las universidades(salvo Brasil).

?? La falta de visibilidad de los aportes por parte de las universidades en el desarrollo de las actividades científicas y tecnológicas tradicionales.

?? La baja formación de docentes con doctorados.

De igual modo, la organización universitaria en relación con la investigación en América Latina, no ha tenido una relevancia en torno a unidades de dirección en términos de vicerrectorías, en la mayoría de ellas, las denominaciones varían en secretarias de investigación, coordinaciones, consejos, direcciones generales y departamentos dependiente en algunos casos de vicerrectorías académicas (México, Argentina ,Chile, Venezuela, etc. ). Y muy pocos casos donde existen vicerrectorías formalmente establecidas ( Costa Rica, Argentina, etc.) encargadas de la gestión de alto nivel estratégico del sistema.

Esta subordinación de la investigación a las decisiones de los órganos académicos influye en la gestión de los procesos del sistema de investigación y en la asignación de recursos al sector, por lo que planificar bajo estas realidades, amerita poca autonomía en las decisiones de áreas críticas claves para el control de gestión como mecanismo válido para el mejoramiento de la dirección integral de estos sistemas.

A manera de reflexión final, el esfuerzo que se ha venido haciendo por parte de las universidades latinoamericanas en torno a la consideración de una praxis gerencial de los sistemas universitarios, es por hoy una realidad en muy pocas de ellas, pero sin embargo, esta idea aglutina muchas esperanzas para el mejoramiento paulatino del sistema en el inicio del presente siglo.

\section{REFERENCIAS Y BIBLIOGRAFÍA}

ABAD, Dario: Control de gestión, Colombia, Interconed Editores, 1997.

AMAT, Joan: Control de gestión(una perspectiva de dirección)Barcelona, España, Edic. Gestión 2000, S.A., 2000.

BELTRAN, Jesús: Indicadores de gestión(herramientas para lograr la competitividad)Colombia, 3R Editores, 1999.

DIAZ, E. y VESSURI, H.: Universidad y Desarrollo Científico-Técnico en América Latina y el Caribe. Caracas, Documento de trabajo № 11, CRESALC-UNESCO, 1982.

GONZALEZ, L. y Hernán Ayarza: Calidad, evaluación institucional y acreditación en la educación superior en la región Latinoamericana y del Caribe. Caracas ,CRESALC/UNESCO, 1997. 
KOONTS, H. y Weihrich. h.: Administración: Una perspectiva Global. México, McGRAW-HILL, 1989.

LEMASSON, J. y Marta Chiappe: La investigación universitaria en América Latina Caracas, Ediciones IESALC/UNESCO, 1999.

LICHA, Isabel(compiladora) Capacidad de Gestión de Centros de Investigación en Venezuela. Caracas, Universidad Central de Venezuela, 1993.

PEÑA C. Jesús : Organizaciones que aprenden(Evaluación de centros de investigación y desarrollo)Caracas, CENDES, 1998.

PEREZ, Juan F.: Control de la gestión empresarial, España, Editorial ESIC, 2000.

ROYERO, Jaim: Contexto mundial sobre la evaluación en las instituciones de educación superior Revista Iberoamericana de Educación [Revista en línea], Disponible: htpp://www.campus-oei.org. 2002.

SERNA, Humberto: Gerencia Estratégica,Caracas, Ediciones Global S.A., 2000.

TUNNERMANN, Carlos: Universidad y Sociedad (Balance histórico y perspectivas desde Latinoamérica) Caracas, Comisión de estudios de postgrado, UCV, /Ministerio de Educación, Cultura y Deportes, 2000.

VESSURI, Hebe(Compiladora): Ciencia Académica en la Venezuela Moderna, Caracas, Fondo Editorial Acta Científica Venezolana, 1984.

VESSURI, Hebe: La calidad de la investigación en Venezuela.(Elementos para el debate en torno al programa de promoción del investigador) Caracas, Interciencia,21(2): 98-102

YERO, Lourdes: "La gestión de la investigación científica en las universidades: ¿Una nueva ilusión? Consideraciones en torno a una gestión de la investigación en la UCV." Pág. 97-142, en Capacidad de Gestión de Centros de Investigación en Venezuela. Caracas, Universidad Central de Venezuela, 1993 


\title{
Contactar
}

Revista lberoamericana de Educación

\author{
Principal OEI
}

